VARIN, Mélanie, CHARBOTEL, Barbara, PEROL, Olivia, PERRIER, Lionel, MASSARDIER-PILONCHERY, Amélie, BONNAND, Sandrine, BELLADAME, E, FORT, Emmanuel, AVRILLON, Virginie, REBATTU, Paul, PEROL, Maurice, FERVERS, Béatrice, 2017, Évaluation d'un auto-questionnaire de repérage des expositions professionnelles chez les patients atteints de cancer bronchopulmonaire, Bulletin du Cancer, 6, 104, Elsevier, pp. 559-564, DOI: 10.1016/j.bulcan.2017.03.014

\title{
Evaluation d'un auto-questionnaire de repérage des expositions professionnelles chez les patients atteints de cancer bronchopulmonaire
}

\section{Assessment of a self-administered questionnaire identifying occupational exposures among lung cancer patients}

\author{
Mélanie Varin ${ }^{1}$, Barbara Charbotel ${ }^{1,2,3}$, Olivia Pérol ${ }^{2}$, Lionel Perrier ${ }^{4}$, Amélie Massardier- Pilonchéry ${ }^{1,3}$, Sandrine \\ Bonnand $^{5}$, Elodie Belladame ${ }^{2}$, Emmanuel Fort ${ }^{1}$, Virginie Avrillon ${ }^{6}$, Paul Rebattu ${ }^{6}$, Maurice Pérol ${ }^{6}$, Béatrice Fervers ${ }^{2}$ \\ ${ }^{1}$ Univ Lyon, Univ Lyon 1, IFSTTAR, UMRESTTE, UMR_T9405, F-69373 LYON, France \\ ${ }^{2}$ Centre Léon- Bérard, Département Cancer et Environnement, 28 rue Laënnec, 69373 Lyon cedex 08, France \\ ${ }^{3}$ Hospices Civils de Lyon, Centre Hospitalier Lyon Sud, Service de pathologies professionnelles, 69495 Pierre- \\ Bénite, France \\ ${ }^{4}$ Univ Lyon, Centre Léon Bérard, GATE L-SE UMR 5824, F-69008 Lyon, France \\ ${ }^{5}$ Centre Léon- Bérard, service social - département des soins de support en oncologie, 28 rue Laënnec, 69373 \\ Lyon Cedex 08, France \\ ${ }^{6}$ Centre Léon- Bérard, département de médecine, 28 rue Laënnec, 69373 Lyon Cedex 08, France
}

\section{Résumé.}

Dix à $29 \%$ des cancers broncho-pulmonaires (CBP) seraient d'origine professionnelle et $60 \%$ d'entre eux ne seraient pas indemnisés.

Le projet PROPOUMON vise à améliorer le repérage, la reconnaissance et l'indemnisation des CBP d'origine professionnelle en maladie professionnelle à partir d'un auto-questionnaire (AQREP). Un des objectifs était d'évaluer la capacité de l'AQREP à détecter les patients pouvant bénéficier d'une consultation " cancers professionnels », en le comparant avec le questionnaire établi par la Société de Pneumologie de Langue Française (Q-SPLF). De Mars 2014 à Septembre 2015, 90 patients pris en charge au Centre Léon Bérard (CLB) pour un CBP ont répondu à l'AQREP et au Q-SPLF. Les deux médecins responsables de la consultation évaluaient ensuite de façon indépendante, sur la base de I'AQREP ou du Q-SPLF, I'indication d'une consultation. Si à l'issue de la consultation des arguments en faveur d'une origine professionnelle étaient réunis, une démarche de déclaration était proposée au patient. L'analyse des questionnaires par les deux médecins était concordante à $73 \%$. L'AQREP a une sensibilité de $72 \%$ et une spécificité de $73 \%$. Ses valeurs prédictives positive et négative sont de $62 \%$ et $82 \%$ respectivement. Les informations renseignées par 24 patients étaient discordantes entre les questionnaires. Pour deux patients ayant une évaluation discordante de l'indication de consultation (AQREP+/Q-SPLF- ; AQREP-/Q-SPLF+), un Certificat Médical Initial a été rédigé. Le premier a été refusé par l'Assurance Maladie, le second est en cours d'instruction. Cette étude a permis de conclure en la capacité de l'AQREP à repérer les CBP potentiellement d'origine professionnelle. Une réunion de concertation pour discuter des cas complexes pourrait être envisagée. Le projet s'élargit actuellement à d'autres centres (Lille, Clermont- Ferrand, Marseille, Caen, Institut St. Catherine Avignon) et aux 
lymphomes.

Mots clés : cancer bronchopulmonaire, exposition professionnelle, auto-questionnaire, maladie professionnelle

\begin{abstract}
.
Ten to $29 \%$ of lung cancers might be linked to occupational factors but $60 \%$ of them are not compensated.

The PROPOUMON project aimed to improve the identification, recognition and compensation of occupational lung cancer as occupational disease using a self-administered questionnaire (AQREP).

One objective was to assess the AQREP, comparing it with the questionnaire drawn up by the French Language Pneumology Society (Q-SPLF). From March 2014 to September 2015, 90 lung cancer patients treated at the Centre Léon Bérard (CLB) responded to the AQREP and Q-SPLF. The two physicians in charge of the consultation assessed independently whether or not a consultation was indicated. A certificate for the compensation process was proposed to the patient when a suspicion of high or average imputability was identified. Analysis of the questionnaires was concordant for $73 \%$ of the patients. The AQREP has a sensitivity of $72 \%$ and a specificity of $73 \%$. Its positive and negative predictive values were $62 \%$ and $82 \%$ respectively. The information provided by 24 patients were discordant between questionnaires. In two patients with discordant evaluation of the indication of consultation (AQREP+/Q-SPLF- ; AQREP-/Q-SPLF+), one Initial Medical Certificate (IMC) was written. This study made it possible to conclude that AQREP is relevant for the identification of potentially occupational lung cancers.

Collegial discussion of complex cases might be considered. The project is currently been extended to other centers (Lille, Clermont-Ferrand, Marseille, Caen, Institute Ste Catherine Avignon) and to lymphoma.
\end{abstract}

Key words : lung cancer, occupational exposure, self-administered questionnaire, occupational disease

\title{
INTRODUCTION :
}

En France et dans les autres pays développés, le cancer broncho-pulmonaire (CBP) représente la 1ère cause de décès par cancer chez les hommes et chez les femmes [1;2]. Bien que le tabac soit reconnu comme le principal facteur de risque de CBP [3], d'autres facteurs de risque, notamment professionnels peuvent être incriminés et seraient responsables de 10 à $29 \%$ des cas des CBP par an [4 -6]. De ce fait, le nombre de CBP attribuables à une origine professionnelle serait comprise entre 2 000 et 4000 cas/ an [7;8]. Parmi ces cas, l'amiante est l'agent le plus fréquemment en cause [7;9]. 
Le CBP peut être indemnisé au titre des maladies professionnelles (MP). Parmi les deux listes officielles de tableaux, 13/114 tableaux du Régime Général (RG) et 5/59 tableaux du Régime Agricole (RA) reconnaissent le CBP comme MP [10]. Dans les cas où le CBP ne remplit pas les conditions du tableau, la reconnaissance du caractère professionnel est subordonnée au système complémentaire de réparation des maladies professionnelles qui repose sur l'avis d'un Comité Régional de Reconnaissance des Maladies Professionnelles (CRRMP).

Alors que la fréquence d'indemnisation en MP des CBP était inférieure à 0.5\% en 1995 [11], elle est passée à $3 \%$ en 2009, soit 1213 cas indemnisés, ce qui reste bien inférieur aux 10 à $29 \%$ des cancers estimés d'origine professionnelle [12]. Entre 2009 et 2013, 85\% des CBP reconnus comme maladies professionnelles l'étaient au titre du tableau 30bis, concernant l'exposition à l'amiante.

Depuis 2009, afin d'améliorer le repérage des CBP d'origine professionnelle et leur indemnisation en maladie professionnelle, le Centre de lutte contre le cancer Léon- Bérard (CLB), en collaboration avec le Centre de consultation de pathologie professionnelle (CCPP) des Hospices Civils de Lyon, a mis en place une démarche de repérage systématique des expositions professionnelles [13]. Ce dispositif comprend la collecte de données professionnelles par un auto-questionnaire (AQREP) permettant de déterminer si le patient doit se voir proposer un rendez-vous pour une consultation dédiée aux cancers professionnels.

Une évaluation préliminaire de ce dispositif a été menée en 2010-2011. Les résultats ont confirmé l'importance des expositions professionnelles chez les patients atteints de CBP et ont permis d'augmenter de façon substantielle les démarches de reconnaissance en MP chez les patients pris en charge au CLB [13]. Par ailleurs, le repérage préalable par l'AQREP avait permis de réduire de près de la moitié les patients à voir en consultation en comparaison à une consultation systématique. II paraissait cependant nécessaire de s'assurer que ce questionnaire n'excluait à tort un patient potentiellement exposé.

Un des objectifs du projet PROPOUMON était d'évaluer la capacité de l'auto-questionnaire (AQREP) à repérer les patients exposés à des cancérogènes professionnels et pouvant bénéficier d'une consultation « cancers professionnels » en le comparant avec le questionnaire établi par la Société de Pneumologie de Langue Française (Q-SPLF), considéré comme un questionnaire de référence par les professionnels.

\section{MATERIEL ET METHODES :}

L'étude PROPOUMON était proposée à tous les patients atteints d'un CBP histologiquement confirmé et pris en charge au CLB entre le 3 Mars 2014 et le 7 septembre 2015, cas incidents sur la période ou traités antérieurement au CLB ou en dehors du CLB. Les patients recevaient l'AQREP par courrier accompagné d'une note d'information et d'une enveloppe $T$ pour le retour du questionnaire. En 
absence de réponse 3 semaines après l'envoi du questionnaire, les patients étaient recontactés de façon systématique par téléphone par une Technicienne de Recherche Clinique (TRC).

\section{Inclusion des patients dans la phase d'évaluation de l'AQREP}

Le sous-échantillon de patients participant à la phase d'évaluation a été constitué à partir des premiers patients inclus dans Propoumon. Ceux-ci ont reçu une note d'information adaptée, les informant d'une participation à deux questionnaires (AQREP et Q-SPLF). Les patients ayant retourné l'AQREP étaient ensuite contactés par téléphone par un interne de médecine et santé au travail pour répondre au questionnaire Q-SPLF. Les entretiens étaient réalisés avec le patient ou pour certains, aidés par l'entourage en cas de difficulté. Cette note d'information spécifique a été envoyée jusqu'à réception de 80 AQREP complétés.

\section{Recueil des données}

Les informations recueillies par le questionnaire de repérage AQREP concernaient les données administratives, le niveau d'étude, l'historique professionnel complet (période militaire, emplois occupés, tâches (habituelles et occasionnelles) effectuées pour chaque emploi, durée, nom, adresse, activité et nombre de salariés de l'entreprise) et une liste de cancérogènes pulmonaires. Cette liste non limitative était établie à partir des nuisances prises en charge au titre des tableaux de maladie professionnelle et de la classification du Centre International de Recherche sur le Cancer (CIRC). Les patients indiquaient s'ils pensaient avoir été exposés ou non à un ou plusieurs des agents listés et renseignaient le poste associé à cette exposition.

Des questions avaient préalablement été ajoutées sur le modèle existant du Q-SPLF pour couvrir des expositions à des cancérogènes pulmonaires avérées, classés après 2002 (gaz d'échappement Diesel par exemple).

\section{Evaluation des questionnaires}

Les deux médecins responsables de la consultation évaluaient ensuite de façon indépendante, pour chaque patient (I'un analysant l'AQREP, l'autre le Q-SPLF), l'indication d'une consultation cancers professionnels. Afin de réduire la subjectivité liée à l'évaluation, les deux médecins ont analysé chacun 50\% des AQREP et 50\% des Q-SPLF. Un algorithme décisionnel a été élaboré.

L'indication de consultation était retenue s'il était identifié une exposition déclarée par le patient à un cancérogène pulmonaire et/ou des emplois et tâches pouvant y être associés. En cas de discordance d'évaluation entre les deux questionnaires, une consultation était proposée au patient, celle-ci étant alors réalisée par le médecin ayant posé l'indication. Lors de la consultation spécialisée, l'entretien permettait de retracer plus précisément le parcours professionnel, compléter les éventuelles expositions à des cancérogènes et identifier les facteurs de risque extraprofessionnels. A l'issue de la 
consultation, lorsque des arguments en faveur d'une origine professionnelle étaient réunis, une démarche de déclaration était proposée au patient. Par ailleurs, lorsque le médecin retrouvait une exposition à l'amiante, une documentation sur la procédure d'indemnisation du Fonds d'Indemnisation des Victimes de l'Amiante (FIVA) lui était remise. Enfin, aux patients pour lesquels un Certificat Médical Initial avait été remis, un accompagnement par une assistante sociale était systématiquement proposé pour se faire aider dans la démarche.

L'issue des déclarations faites par les patients était connue sur demande auprès des patients, de la Caisse Primaire d'Assurance Maladie et du FIVA.

\section{Analyse des données}

La sensibilité et la spécificité c'est-à-dire la capacité du questionnaire AQREP à détecter respectivement les patients positifs et les patients négatifs par rapport au questionnaire SPLF ont été évaluées à partir de la procédure Freq du logiciel SAS, version 9.3.La valeur prédictive positive c'est-àdire la proportion de patients ayant une indication de consultation déterminée à partir de l'évaluation de l'AQREP et de l'évaluation du Q-SPLF ainsi que la valeur prédictive négative c'est-à-dire la proportion de patients n'ayant pas d'indication de consultation suite à l'évaluation de l'AQREP et de l'évaluation du Q-SPFL sont également calculées.

\section{Analyse des divergences}

Une analyse plus qualitative des divergences entre les deux évaluateurs a été réalisée par l'équipe en comparant, dossier par dossier, les écarts entre questionnaires et entre évaluateurs.

\section{RESULTATS}

De Mars 2014 à Septembre 2015, 440 patients pris en charge au CLB pour un CBP ont été inclus dans l'étude PROPOUMON. Au total, 53\% des 440 patients inclus ont retourné I'AQREP. La majorité des patients l'a retourné dans un délai supérieur à 3 semaines $(69 \%, 161 / 234)$ et $24 \%$ après relance téléphonique.

L'étude d'évaluation de l'AQREP a été proposée auxpremiers patients ayant répondu à l'AQREP dans le cadre de l'étude Propoumon, jusqu'à atteindre l'effectif de 80 patients. Le nombre de retour d'AQREP complétés ne pouvant être définis en amont, 90 patients ont finalement été inclus dans cette étude d'évaluation. Aucun patient n'a refusé de répondre à l'entretien téléphonique pour renseigner le questionnaire SPLF. Un patient ayant complété l'AQREP mais dont le Q-SPLF a été rempli par son épouse, a été exclu de l’analyse car il était nécessaire pour évaluer les discordances entre questionnaires que ceux-ci soient renseignés par la même personne. Au total, 89 patients ont été retenus dans la présente analyse.

\section{Analyse des questionnaires}


Les 178 questionnaires des 89 patients (89 AQREP / 89 Q- SPLF) ont été analysés par les deux médecins responsables du projet. Pour chaque patient, un médecin analysait l'AQREP tandis que l'autre analysait le Q- SPLF.

L'analyse des questionnaires par les deux médecins était concordante à 73\%. Parmi les 65 analyses concordantes, 24 étaient en faveur d'une consultation et 41 concluaient à l'absence d'indication de consultation, sur la base des informations renseignées par le patient. En revanche, pour 24 patients (27\%), I'analyse par les deux médecins évaluateurs était discordante (15 AQREP+/Q-SPLF - ; 9 AQREP(Q-SPLF +).

En conséquence, l'AQREP a une sensibilité de $72 \%$ et une spécificité de $73 \%$ par rapport au Q-SPLF. Sa valeur prédictive positive est de $62 \%$ et sa valeur prédictive négative de $82 \%$.

L'analyse rétrospective des 24 décisions discordantes a retrouvé plusieurs causes. Si $54 \%$ des discordances peuvent s'expliquer par une évaluation divergente entre les deux médecins, pour les autres questionnaires $(\mathrm{N}=11)$ l'analyse qualitative a révélé que la différence en terme d'évaluation était principalement due à des informations différentes recueillies par les questionnaires $(N=6)$ ou au fait que les patients avaient transmis des informations divergentes $(\mathrm{N}=5)$.

\section{Consultations}

Au total, une indication de consultation a été posée suite à l'analyse de l'AQREP et /ou du Q-SPLF pour 48 des patients (54\%). Parmi les 48 consultations proposées, 39 patients (81\%) ont été vus en consultation "cancers professionnels". Concernant les neuf patients n'ayant pas été vus en consultation, 3 étaient décédés, 2 ont refusé, 1 présentait une altération de l'état général trop importante et pour 3 la raison n'est pas connue, les patients ne s'étant pas présenté suite à la proposition de rendez-vous.

\section{Démarche de reconnaissance en MP}

A l'issue des 39 consultations réalisées, $14 \mathrm{CMI}$ ont été rédigés (35\%) (Figure 1), dont 11 au titre du tableau 30bis « Cancer broncho-pulmonaire provoqué par l'inhalation de poussières d'amiante ». Les documents nécessaires à la demande d'indemnisation par le FIVA ont également été remis à ces 11 patients. Deux CMI ont été rédigés pour une pathologie potentiellement indemnisable, hors tableau (l'un pour tabagisme passif et l'autre pour une exposition aux peintures pendant deux ans et à l'amiante d'une durée inférieure à 2 ans, ce dernier CMI ayant été rédigé à la demande du patient). Sur les 24 évaluations discordantes, un CMI a été rédigé pour deux patients, dont l'un (AQREP+/QSPLF-) à la demande du patient. Cette demande de reconnaissance en MP a été refusée par l'Assurance Maladie. Pour l'autre CMI (AQREP-/Q-SPLF+), la demande est actuellement en cours d'instruction par 
I'Assurance Maladie. L'issue des demandes de reconnaissance en pathologie professionnelle est détaillée dans la Figure 1.

\section{DISCUSSION :}

L'objectif de notre étude était d'évaluer une démarche systématique et individuelle de recherche des expositions professionnelles chez les patients atteints de CBP, à partir d'un auto-questionnaire de repérage des expositions professionnelles (AQREP).

Une première évaluation de ce dispositif avait été menée au CLB en 2010-2011, confirmant la fréquence d'expositions professionnelles chez les patients atteints de CBP et ayant permis d'augmenter leur reconnaissance en pathologie professionnelle [13]. Les résultats de notre étude actuelle sont les premiers à évaluer cette démarche, en comparant l'AQREP au Q-SPLF [14]. Ce questionnaire, très complet, résultat d'une action commune des sociétés françaises de pneumologie et médecine du travail, a été considéré comme une référence pour les professionnels malgré l'absence de démarche de validation publiée. Destiné aux pneumologues et mis en place à partir de 2002, il doit être administré par un médecin lors d'une consultation [15]. L'auto-questionnaire (AQREP), développé pour le repérage des expositions professionnelles au CLB, est plus synthétique et centré sur l'objectif suivant : identifier les patients à voir en consultation spécialisée sur la base de l'analyse de l'histoire professionnelle décrite par le patient (incluant les tâches effectuées) et d'une exposition déclarée par le patient à un cancérogène pulmonaireà partir d'une liste ,. L'objectif de la consultation est alors de faire préciser les conditions d'expositions. L'intérêt de l'autoquestionnaire envoyé par courrier est d'être systématique et de donner à tous les patients l'opportunité d'être informés de leurs droits. II convient cependant de s'assurer que la consultation est bien proposée à tous les patients qui peuvent bénéficier d'une indemnisation. C'est donc la valeur prédictive négative qui est le critère essentiel à atteindre. Elle est ici de $82 \%$ sur la base des comparaisons entre questionnaires. Elle est en réalité supérieure puisqu'un seul patient repéré uniquement par le Q-SPLF semblait relever potentiellement d'une indemnisation dont l'issue reste en attente. De plus, II est important de prendre en compte les raisons qui ont conduit à des discordances dans l'analyse des questionnaires. En effet, une discordance a été observée entre les 2 questionnaires pour 24 patients (27\%). Or, pour 11 de ces patients, le contenu de l'information recueilli par les deux questionnaires était différent $(n=6)$, ou le patient fournissait des informations discordantes sur les 2 questionnaires $(n=5)$. En effet, dans l'AQREP, la recherche d'une exposition professionnelle se fait d'une part par le détail du parcours professionnel du patient et d'autre part par la recherche de cancérogènes pulmonaires. La description précise du poste avec les tâches effectuées, les techniques et produits utilisés, la durée, ainsi que le nom et l'activité de l'entreprise sont autant d'éléments permettant au médecin d'évaluer si le patient a été exposé ou non à un cancérogène pulmonaire. En revanche, le Q-SPLF, ne recueille pas d'informations 
concernant le détail du parcours professionnel ni des postes occupés. Le patient répond par oui ou non à une liste de métiers proposés par le médecin. Si la réponse est positive, il indique alors la durée d'exposition pour cet emploi. Cette démarche restrictive a parfois conduit l'interrogateur à mener un interrogatoire plus dirigé. Le patient pouvait être interrogé plus précisément sur les tâches effectuées ou bien, lorsqu'aucun des métiers n'était retenu à la fin du questionnaire, le médecin demandait au patient de lister de lui-même les emplois occupés. Ainsi, prenons l'exemple d'un patient qui avait bien détaillé les tâches effectuées à son poste d'aide-soignant dans I'AQREP. Cette description a permis à l'évaluateur de suspecter une exposition aux rayonnements ionisants, inconnue du patient. Dans le QSPLF, la question de l'exposition aux rayonnements ionisants étant posée directement, le patient y a répondu par la négative. Ces discordances peuvent expliquer en partie les différences d'évaluation entre les 2 médecins. Par ailleurs, l'analyse rétrospective des 13 dossiers pour lesquels les discordances étaient liées à une évaluation divergente des deux médecins sur la base des mêmes informations fournies dans les deux questionnaires, a permis de mettre en évidence des critères différents pour poser l'indication de consultation. Ainsi, l'un des médecins privilégiait la possibilité de déclaration en maladie professionnelle à l'issue de la consultation tandis que l'autre prenait en compte le profil du patient (par exemple, patient jeune, non-fumeur) ainsi que l'exposition à des substances cancérogènes probables (même sans tableau existant du Régime Général). Au final, ce médecin proposait plus souvent des consultations sans qu'elles n'aboutissent à une proposition d'effectuer les démarches de reconnaissance en pathologie professionnelle. Considérant cette différence d'évaluation entre les deux médecins, de spécialité différente, il serait pertinent de mettre en place au sein du CLB des réunions de concertation entre les évaluateurs afin de discuter des cas complexes.

\section{CONCLUSION :}

Le recueil de données professionnelles par auto-questionnaire mis en place par le CLB en collaboration avec le CCPP des Hospices Civils de Lyon semble pertinent pour identifier les patients susceptibles de relever d'une indemnisation en MP. Lauréat du Prix de l'Innovation d'Unicancer dans la catégorie «Prévention et Dépistage » en 2014, le projet PROPOUMON s'inscrit directement dans le Plan Cancer 2014-2019 [19]. II répond à la mesure 12.3 «Améliorer l'identification des cancers d'origine professionnelle pour permettre leur reconnaissance en maladie professionnelle ».

Les résultats du présent projet soulignent l'intérêt d'améliorer et d'étendre cette démarche à d'autres pathologies cancéreuses associées à une exposition à des cancérogènes professionnels. Actuellement, une démarche similaire est mise en œuvre pour les lymphomes conjointement au CLB et au Centre Hospitalier Lyon Sud, centres de référence pour le traitement de cette pathologie.

Compte tenu du taux important de non-réponse et des enjeux médico-sociaux de la reconnaissance des cancers d'origine professionnelle, il est important de renforcer ces dispositifs d'information; 
l'objectif étant d'améliorer le repérage des expositions professionnelles pour les professionnels de santé et de pouvoir faire un choix en étant mieux informés sur la démarche de reconnaissance pour les patients.

Grâce à la validation de l'AQREP, le projet PROPOUMON est pérennisé et s'élargit à d'autres établissements (Institut Sainte Catherine à Avignon) et Centres de Lutte contre le Cancer candidats (Lille, Clermont- Ferrand, Marseille et Caen) dans le cadre d'une démarche multicentrique.

Financement : le projet Propoumon a bénéficié d'un financement de I'INCa DEPREV 2013-147 et de la Chaire d'excellence " environnement, nutrition et cancer » du cancéropole Lyon- Auvergne-Rhône-Alpes avec le soutien du laboratoire Merck-Serono.

Conflits d'intérêts : aucun

\section{BIBLIOGRAPHIE}

1. Torre LA, Bray F, Siegel RL, Ferlay J, Lortet-Tieulent J, Jemal A. Global Cancer Statistics, 2012. CA Cancer J Clin. 2015;65:87-108.

2. E-cancer.fr [Internet]. Boulogne Billancourt : Institut National du Cancer [consulté le 14/11/2015]. Incidence et mortalité nationales du cancer du poumon en 2015. Disponible sur : http://lesdonnees.e-cancer.fr/les-fichesde-synthese/1-types-cancer/12-cancer-poumon/30-incidence-mortalite-france-cancer-poumon.html

3. Islami F, Torre LA, Jemal A. Global trends of lung cancer mortality and smoking prevalence. Trans Lung Cancer Res. 2015 Aug;4(4):327-38.

4. Cogliano VJ, Baan R, Straif K, Grosse Y, Lauby-Secretan B, El Ghissassi F, et al. Preventable exposures associated with human cancers. J Natl Cancer Inst 2011 Dec 21;103(24):1827-39.

5. Pairon JC, Brochard P, Le Bourgeois JP, Ruffié P. Les cancers professionnels. Tome I. Paris : Margaux- Orange, 2000

6. Massardier- Pilonchery A, Charbotel B, Normand JC, Bergeret A. Cancers professionnels. EMC - Pathologie professionnelle et de l'environnement 2013;8:1-12 (article 16-532- A- 10)

7. Imbernon E. Estimation du nombre de cas de certains cancers attribuables à des facteurs professionnels en France. Saint Maurice : Institut de Veille Sanitaire; Sep 2002. 28 p.

8. Boffetta $P$, Autier P, Boniol M, Boyle P, Hill C, Aurengo A, Masse R, et al. An estimate of cancers attributable to occupational exposures in France. J Occup environ med / 2010 Apr;52(4):399-406.

9. Gilg Soit IIg A, Houot M, Audignon-Durand S, Brochard P, El Yamani M, Imbernon E, et al. Estimation des parts attribuables de cancers aux expositions professionnelles à l'amiante en France : utilisation des matrices développées dans le cadre du programme Matgéné. Bull épidémiol hebd [Internet]. Jan 2015 [consulté le 13/10/2015];3-4:[7 p.]. Disponible sur : http://invs.santepubliquefrance.fr/beh/2015/3-4/2015 3-4 6.html

10. Institut national de recherche et de sécurité. Les maladies professionnelles. Guide d'accès aux tableaux de Régime général et du Régime agricole de la Sécurité sociale. 9è ed. Paris : INRS; 2012. p. 349.

11. De Lamberterie G, Maitre A, Goux A, Brambilla C, Perdrix A. Sous-déclaration des cancers bronchiques professionnels. À propos d'une expérience pilote au CHU de Grenoble de 1996 à 1998. Rev Mal Respir. 2002;19(2):190-5.

12. Risquesprofessionnels.ameli.fr [Internet]. Paris : Caisse nationale de l'Assurance maladie des travailleurs salariés [consulté le 25.09.2015]. Rapport de gestion 2012. Disponible sur:

http://www.risquesprofessionnels.ameli.fr/fileadmin/user upload/document PDF a telecharger/brochures/R apport gestion 2012.pdf

13. Cellier C, Charbotel B, Carretier J, Rebattu P, Fayette J, Pérol M, et al. Repérage des expositions professionnelles chez les patients atteints de cancers bronchopulmonaires. Bull Cancer. Juil-Août 2013;100(78):661-70.

14. Société de pneumologie de langue française et Société française de médecine du travail. Questionnaire de repérage des expositions professionnelles chez les sujets atteints de cancer bronchique primitif. Info Respiration 2002 ; (47) : 13-22. www.splf.org/s/IMG/pdf/questCMP-2.pdf

15. Dalphin JC. Réduire la sous déclaration en maladie professionnelle des cancers bronchiques : une action SPLFSFMT. Rev Mal Respir. Avr 2002;19(2):149-51. 
16. Legrand Cattan K, Chouaid C, Monnet I, Atassi K, Bassinet L, Dhissi G et al. Evaluation des expositions professionnelles et cancer broncho-pulmonaire. Rev Mal Respir. 2000;17:957-62.

17. Normand JC, Bergeret A, Gérinière L, Souquet PJ, Prost G. Six années d'expérience d'interrogatoire systématique en vue de la reconnaissance des cancers bronchopulmonaires : bilan, limites et enseignements. XXVle congrès national de médecine du travail, Lille. Le risque cancérogène : du repérage à la surveillance médicale. Arch Med Prof 2001;62:186-7.

18. Perrier L, Nechba A, Perol O, Perol M, Fayette J, Rebattu P, et al. Cost of Questionnaire-Based Process to Identify Occupational Exposures Among Patients with Lung Cancer. Value Health. 2015 Nov;18(7):A485.

19. E-cancer.fr [Internet]. Boulogne Billancourt : Institut National du Cancer [consulté le 03.03.16]. Plan cancer 2014-2019. Disponible sur : http://www.e-cancer.fr/Expertises-et-publications/Catalogue-despublications/Plan-Cancer-2014-20192. 




Figure 1 Etapes et résultats du repérage des expositions professionnelles chez les patients atteints de cancer bronchopulmonaire primitif. CMI : certificat médical initial ; FIVA : Fonds d'Indemnisation des Victimes de l'Amiante 